\title{
METÁSTASIS CUTÁNEA DE ADENOCARCINOMA DE CUELLO UTERINO. REPORTE DE CASO
}

\section{CUTANEOUS METASTASIS OF CERVIX ADENOCARCINOMA. CASE REPORT}

\section{Evelyn CUEVAS JARA ${ }^{1}$, Beatriz DI MARTINO ORTIZ², Mirtha RODRÍGUEZ MASI ${ }^{3}$, Oilda KNOPFELMACHER ${ }^{3}$, Lourdes BOLLA DE LEZCANO 4 .}

${ }^{1}$ Médica residente de Dermatología, Facultad de Ciencias Médicas, Universidad Nacional de Asunción, San Lorenzo - Paraguay.

${ }^{2}$ Profesora Asistente de Dermatología, Facultad de Ciencias Médicas, Universidad Nacional de Asunción, San Lorenzo - Paraguay.

${ }^{3}$ Profesora Titular de Dermatología, Facultad de Ciencias Médicas, Universidad Nacional de Asunción, San Lorenzo - Paraguay.

${ }^{4}$ Profesora Titular y Jefa de la Cátedra de Dermatología, Facultad de Ciencias Médicas, Universidad Nacional de Asunción, San Lorenzo - Paraguay.

Cómo citar este artículo: Cuevas Jara E, Di Martino Ortiz B, Rodríguez Masi M, Knopfelmacher O, Bolla de Lezcano L. Metástasis cutánea de adenocarcinoma de cuello uterino. Reporte de caso. Medicina Clínica y Social. 2017;1(2): 143-149.

\section{RESUMEN}

Las metástasis cutáneas de tumores primarios internos son un evento raro en dermatología. Sin embargo con el aumento en la frecuencia de neoplasias internas, los dermatólogos pueden ser los primeros en descubrir la enfermedad y, tras el tratamiento, las metástasis cutáneas pueden ser el primer signo de recidiva. Presentamos el caso de una paciente adulta con antecedente de adenocarcinoma de cuello uterino con lesiones cutáneas en mama y hemiabdomen derecho, compatible a la histopatología con metástasis cutánea (dérmica) por un adenocarcinoma mucosecretante moderadamente diferenciado. Además realizamos una breve revisión de la literatura.

Palabras clave: Metástasis cutánea; Adenocarcinoma; Cuello uterino.

\section{ABSTRACT}

Cutaneous metastases of primary internal tumors are a rare event in dermatology. However, with the increase in the frequency of internal tumors, dermatologists may be the first ones to discover the disease and, after treatment, cutaneous metastases may be the first sign of relapse. We present the case of an adult female patient with a history of cervix adenocarcinoma with cutaneous lesions in the breast and right hemiabdomen, compatible to the histopathology with cutaneous (dermal) metastasis by a moderately differentiated mucosecreting adenocarcinoma. In addition we make a brief review of the literature.

Keywords: Cutaneous metastases; Adenocarcinoma; Cervix. 


\section{INTRODUCCIÓN}

Se define metástasis cutánea a la neoplasia maligna que compromete la dermis o el tejido subcutáneo, y que no mantiene una relación de contigüidad con el tumor primario que le da origen (1).

Las metástasis cutáneas de tumores primarios internos son un evento raro en dermatología, apareciendo en un 0,7\% - 9\% de pacientes con cáncer. La mayoría ocurren tras el diagnóstico del tumor primario, como una manifestación tardía de la enfermedad diseminada, con un intervalo superior a 5 años en un 7\% - 12\% de los casos entre el diagnóstico del primario y la metástasis. Algunas metástasis ocurren de forma coincidente al primario y unas pocas antes del diagnóstico del mismo $(1,2)$.

Las metástasis cutáneas tienen un importante valor pronóstico dado que reducen sustancialmente la supervivencia (2).

\section{PRESENTACIÓN DEL CASO CLÍNICO}

Mujer, 45 años, ama de casa, procedente de la ciudad de Caacupé (Departamento de Cordilla, Paraguay) con diagnóstico de adenocarcinoma de cuello uterino desde hace 3 años, tratado con cirugía más quimioterapia (última quimioterapia con cisplatino + 5-fluorouracilo, 6 meses antes de la consulta).

Refiere manchas rojas de 1 mes de evolución, que inician en mama derecha, con los días se extienden a hemiabdomen derecho. Las lesiones se sobre elevan y se acompañan de prurito intenso, sin predominio de horario, que empeora con el calor y el sudor. El rascado produce sangrado de las lesiones. Tratada con loción que contiene fenol, alcanfor, difenhidramina, óxido de zinc y neocalamina, sin mejoría.

\section{Examen físico}

Placa eritematosa con centro de coloración no homogénea, bordes irregulares, límites netos, de $25 \times 12 \mathrm{~cm}$, sobre la que asientan múltiples pápulas eritematosas brillantes, redondeadas, bordes regulares, límites netos, algunas con collar de escamas y otras con costras hemáticas de $0,3-0,8 \mathrm{~cm}$ de diámetro, en mama derecha. Placas de similares características de $2-4 \mathrm{~cm}$ localizadas en abdomen, predominio derecho (Figuras 1, 2 y 3 ).

Figura 1. Clínica y localización de las lesiones.

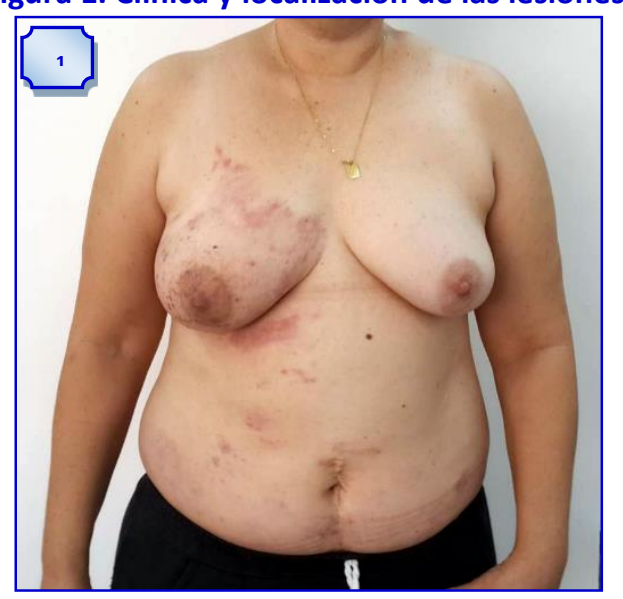


Figuras 2 y 3 . Clínica y localización de las lesiones.

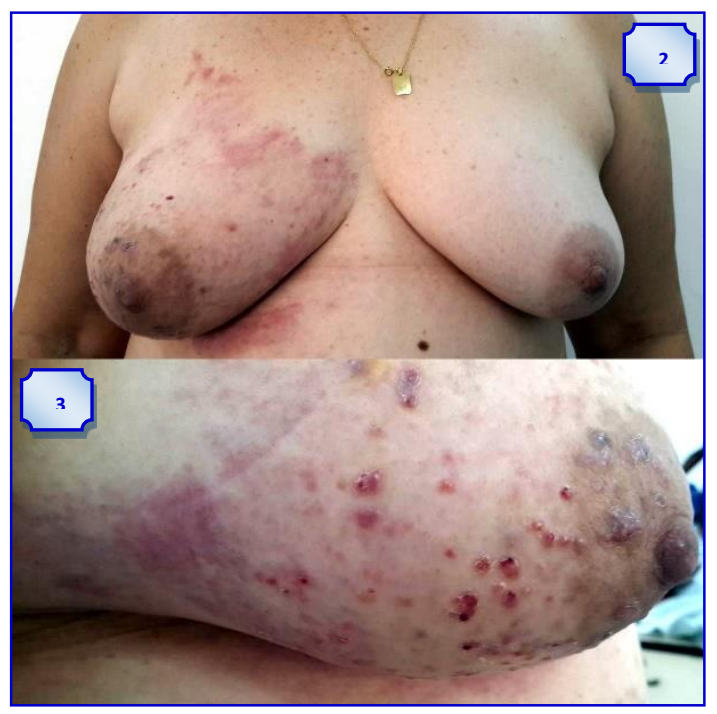

Figuras 1, 2, y 3: Clínica. Placa eritematosa con centro de coloración no homogénea, sobre la que asientan múltiples pápulas eritematosas brillantes en mama derecha. Placas de similares caracteristicas en abdomen, predominio derecho.

\section{Histopatología}

Se observa infiltración dérmica por una proliferación neoplásica de estirpe epitelial que forma luces glandulares, en áreas con cierto patrón cribiforme. Las células que tapizan las glándulas son de morfología cilíndrica, los núcleos son grandes e hipercromáticos, con disrelación núcleo/citoplasma, el citoplasma es basófilo claro con vacuolas de mucosecreción. Hay abundante mucosecreción dentro de las glándulas y también como pools en el tejido de la dermis. No hay infiltración de vasos sanguíneos ni linfáticos por elementos neoplásicos. No hay invasiones perineurales. Tampoco hay afectación epidérmica (Figuras 4, 5 y 6).

Figuras 4, 5 y 6 . Histopatología de las lesiones.

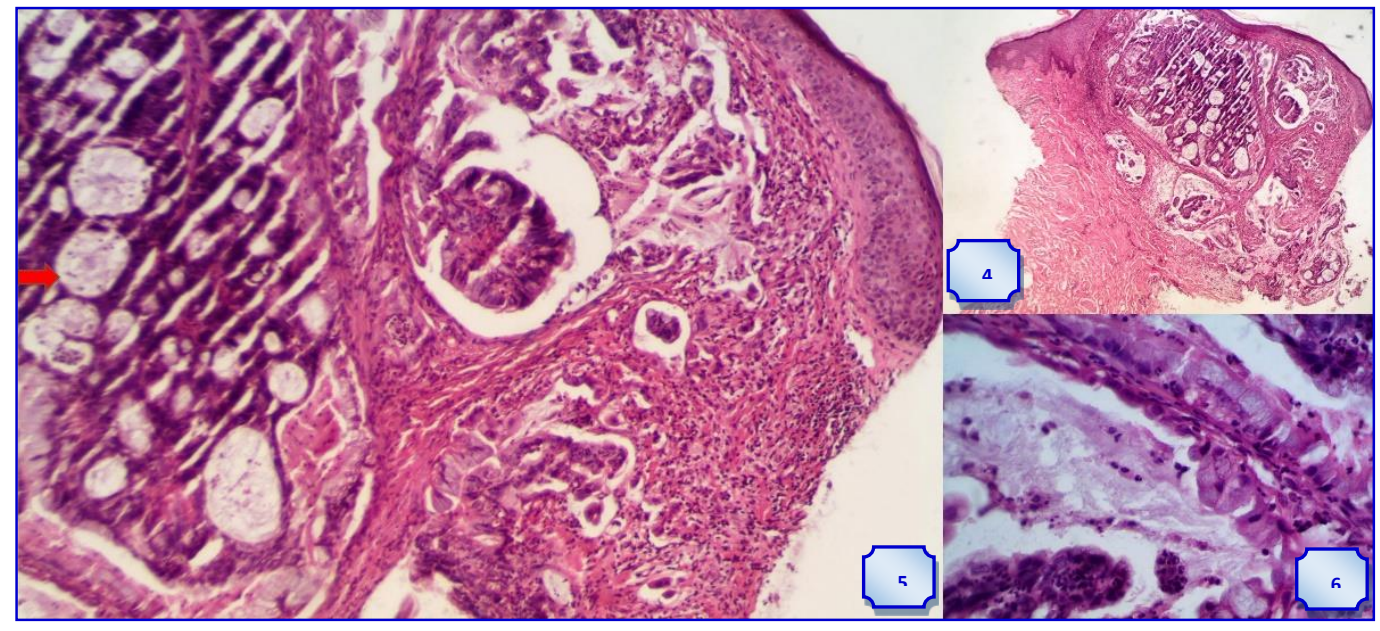

Figura 4: Histopatología. Proceso infiltrante dérmico sin afectación epidérmica (HE 10X). Figura 5: Histopatología. Infiltración dérmica por proliferación neoplásica de estirpe epitelial que forma luces glandulares, en áreas con cierto patrón cribiforme. Hay abundante mucosecreción dentro de las glándulas y también como pools en el tejido de la dermis (HE 40X). Figura 6: Histopatología. Las células que tapizan las glándulas son de morfología cilíndrica, los núcleos son grandes e hipercromáticos, con disrelación núcleo/citoplasma. El citoplasma es basófilo claro con vacuolas de mucosecreción. 


\section{Diagnóstico final}

Metástasis cutánea (dérmica) por adenocarcinoma mucosecretante moderadamente diferenciado. La paciente fue derivada a oncología para continuar tratamiento quimioterapéutico.

\section{DISCUSIÓN}

De las lesiones malignas que se pueden encontrar en piel, hasta el $4 \%$ corresponde a una metástasis y se encuentran ubicadas principalmente en la dermis o tejido celular subcutáneo, y solo en pocos casos invadiendo la epidermis (3). Las metástasis cutáneas son el resultado de la infiltración de la piel por proliferaciones de células procedentes de tumores malignos situados a distancia (4).

\section{Epidemiología}

Las metástasis cutáneas se observan con mayor frecuencia en el sexo femenino y en individuos entre 50 a 70 años. En realidad, la mayoría de los datos epidemiológicos está supeditada a la incidencia o prevalencia de las neoplasias malignas de las cuales se originan y al potencial metastático en piel de las mismas $(1,2,4)$.

Si bien el cáncer de mama es el origen más frecuente de metástasis cutáneas en el ser humano, la neoplasia con mayor potencial metastático a piel es el melanoma. En investigaciones basadas en registros médicos se ha observado que aproximadamente del $10 \%$ al $13 \%$ de los pacientes desarrolla metástasis cutáneas locorregionales o distantes, y que al menos el $8 \%$ de los individuos con enfermedad localizada puede manifestar metástasis en tránsito o satelitosis. Un estudio en autopsias llegó a demostrar la presencia de metástasis cutáneas distantes en el $75 \%$ de los casos analizados. El melanoma se considera la primera causa de metástasis cutáneas en varones y la segunda en mujeres $(1,2,5,6)$.

Otras neoplasias que provocan metástasis cutáneas en la población general son el cáncer de pulmón, el cáncer de mucosa oral, el cáncer de colon, el cáncer de ovario y el cáncer de riñón $(1,2,6,7)$ (Tabla 1).

\begin{tabular}{|c|c|c|c|}
\hline \multicolumn{4}{|c|}{$\begin{array}{c}\text { TABLA 1. FRECUENCIA APROXIMADA DE LAS PRINCIPALES } \\
\text { CAUSAS DE METÁSTASIS CUTÁNEAS SEGÚN EL SEXO }\end{array}$} \\
\hline \multicolumn{2}{|c|}{ Mujeres } \\
\hline
\end{tabular}

Algunas neoplasias que tienen alta prevalencia en la población general pero que muy rara vez provocan metástasis cutáneas son el cáncer de próstata, el cáncer de cuello uterino y el cáncer de hígado $(1,2,6)$.

\section{Etiopatogenia}


Las metástasis surgen cuando las células neoplásicas se desprenden de un tumor primario y se diseminan a otros lugares. El mecanismo de producción de las metástasis es variable, y se han implicado varías vías en su desarrollo. Las vías hematógena y linfática son las más frecuentes, aunque la diferenciación entre ambas es difícil debido a que están interconectadas. La diseminación linfática es la vía más frecuente de propagación inicial de la mayoría de los tumores malignos. La difusión regional se produce más a menudo a través de las cavidades corporales, especialmente la cavidad peritoneal. El trasplante de células tumorales puede producirse por el transporte mecánico de fragmentos tumorales mediante los instrumentos quirúrgicos durante la cirugía u otros procedimientos invasivos, pero esto rara vez ocurre $(1,2,4)$.

\section{Variantes clínicas}

Las metástasis cutáneas pueden adoptar morfologías muy variadas.

La forma nodular es la más frecuente, y sus lesiones, únicas o múltiples, suelen ser indoloras. Su coloración es variable, habitualmente eritematosa o incluso color piel normal. Con frecuencia se ulceran. Prácticamente todos los tumores que ocasionan metástasis cutáneas pueden presentarse de esta manera $(1,2,5,7)$. El cáncer de riñón suele manifestarse por metástasis nodulares de aspecto angiomatoide y fácil sangrado, que remeda al botriomicoma, al sarcoma de Kaposi o a los hemangiomas. Las metástasis cutáneas del carcinoma tiroideo también pueden adoptar características similares (1).

El carcinoma inflamatorio metastático o erisipelatoide se presenta con una placa eritematoedematosa con aumento de temperatura local y que puede doler, indistinguible de la erisipela, salvo por la falta de signos y síntomas de compromiso sistémico. Se asocia principalmente con cáncer de mama y menos frecuentemente de pulmón, colorrectal y melanoma (1, 2, 4).

La forma telangiectática suele presentarse como un área circunscripta de telangiectasias o papulovesículas violáceas. En ocasiones puede adquirir un aspecto contusiforme. Es casi exclusiva del cáncer de mama. Debe realizarse el diagnóstico diferencial con el angiosarcoma y con el linfangioma circunscripto $(1,2,4)$.

La variante en coraza comienza como una placa edematosa que evoluciona con engrosamiento cutáneo, hasta adoptar un aspecto similar al de la morfea. Con el tiempo es común que se agreguen lesiones noduloides y papuloides Se observa en el cáncer de mama y otros tumores malignos como el cáncer de pulmón, riñón y de origen gastrointestinal (1-4).

Existen otras presentaciones menos frecuentes, llamadas atípicas. Entre ellas se describen formas ampollares, zosteriformes, alopécicas, cicatrizales, pseudomixedematosas, entre otras (1).

\section{Localización}

Las metástasis cutáneas tienden a aparecer en proximidad al tumor primario. El cuero cabelludo, el ombligo, la pared torácica y el abdomen son las localizaciones más frecuentes para las metástasis. En el $75 \%$ de los varones las metástasis afectan al cuero cabelludo, cuello, tórax y abdomen. En el $75 \%$ de las mujeres aparecen en el tórax y abdomen $(1-4,6,8)$. 
La pared abdominal es la localización más frecuente de las metástasis cutáneas y hasta en un $10 \%$ se afecta el ombligo, siendo los cánceres gastrointestinales los que metastatizan en esta localización con mayor frecuencia. Otros cánceres que metastatizan a nivel abdominal son el de pulmón, ovario y mama. En la pelvis generalmente metastatizan los tumores de intestino grueso $(1-3,5,7)$. El cuero cabelludo es una localización que atrae a diferentes tipos de metástasis cutáneas. En hombres, con frecuencia los primarios proceden de pulmón o riñón, tendiendo a aparecer como un hallazgo precoz. En mujeres, el primario más frecuente es el cáncer de mama y la metástasis tiende a aparecer de forma tardía $(1,2,4,6,7)$.

Las metástasis faciales generalmente proceden de carcinomas de la cavidad oral, hipernefromas, cánceres de pulmón y de mama. Las metástasis palpebrales tienen como primarios melanomas y cánceres de mama. La afectación del cuello suele aparecer por extensión directa de ganglios cervicales, teniendo los primarios a nivel de cavidad oral, pulmón o mama (1-5). Finalmente, las metástasis en extremidades superiores son raras y generalmente un hallazgo tardío. Son producidas por melanomas y primarios de mama, pulmón, riñón y colon. Las piernas son una localización aún menos frecuente, siendo los primarios más frecuentes los melanomas, el cáncer de pulmón y renal $(1,2,6)$.

\section{Diagnóstico}

El diagnóstico de certeza de las metástasis cutáneas es histológico. En la piel, a nivel histológico, con frecuencia se observan características propias de la neoplasia primaria. Sin embargo, a menudo las metástasis son variantes muy poco diferenciadas del tumor original, y el estudio microscópico puede ser insuficiente para arribar al diagnóstico etiológico. En este contexto, el aporte de la inmunohistoquímica resulta invalorable $(1,2,4)$ (Tabla 2).

TABLA 2. INMUNOFENOTIPOS DE LAS METÁSTASIS DE LOS PRINCIPALES CARCINOMAS

\begin{tabular}{|c|c|}
\hline Tumor Primario & Marcadores inmunohistoquímicos \\
\hline Mama & $\begin{array}{c}\text { CK7 (+), CAM } 5.2(+) \text {, vimentina (-), TTF-1 (-), Ber-EP4 (+), WT-1 (-), } \\
\text { DPC4 (-) }\end{array}$ \\
\hline Adenocarcinoma de pulmón & CK7 (+), CAM $5.2(+)$, CEA (+), Ber-EP4 (+), WT-1 (-), DPC4 (-) \\
\hline Colorrectal & $\begin{array}{c}\text { CK20 (+), CAM } 5.2(+), \text { CK17 (-), CK19 (+), CEA (+), TTF-1 (-), Ber-EP4 } \\
(+), \text { S100 (-), WT-1(-), DPC4 (-) }\end{array}$ \\
\hline Gástrico & $\begin{array}{c}\text { CAM } 5.2(+) \text {, vimentina }(-) \text {, TTF-1 (-), ER }(-) \text {, Ber-EP4 (+), WT-1 (-), } \\
\text { DPC4 (-) }\end{array}$ \\
\hline Próstata & $\begin{array}{l}\operatorname{CK} 7(-), \operatorname{CK} 20(-), \operatorname{CAM} 5.2(+), \operatorname{CD} 5 / 6(-), \operatorname{CK} 17(-), \operatorname{CEA}(-) \text {, } \\
\text { vimentina }(-), \operatorname{TTF}-1(-), \operatorname{ER}(-), \operatorname{Ber}-\operatorname{EP} 4(+), \operatorname{S100}(-), \text { WT-1 }(-), \text { DPC4 (-) }\end{array}$ \\
\hline Páncreas & $\begin{array}{c}\text { CK7 (+), CAM } 5.2(+) \text {, vimentina }(-), \text { TTF-1 }(-) \text {, ER }(-), \text { Ber-EP4 }(+), \\
\text { S100 (-), WT-1 (-), DPC } 4(+)\end{array}$ \\
\hline Renal & $\begin{array}{c}\operatorname{CK} 7(-), \operatorname{CK} 20(-), \operatorname{CAM} 5.2(+), \operatorname{CEA}(-), \operatorname{TTF}-1(-), \text { Ca-125 (-), ER }(-), \\
\text { CD10 (+), WT-1 (-), DPC4 (-) }\end{array}$ \\
\hline Neuroendocrino & CK20 (-), CK5/6 (-), Ca-125 (-), ER (-), Ber-EP4 (-), WT-1 (-), DPC4 (-) \\
\hline Carcinoma de células escamosas & $\begin{array}{c}\text { CK7 (-), CK20 (-), CK5/6 (+), CK17 (+), TTF-1 (-), CA19.9 (-), Ca-125 (-), } \\
\text { ER }(-) \text {, Ber-EP4 }(-), \text { CD10 (-), S100 (-), WT-1 (-), DPC4 (-) }\end{array}$ \\
\hline \multicolumn{2}{|c|}{$\begin{array}{l}\text { (+) indica «siempre positivo" y (-) «negativo, con raras excepciones». Ber-EP4: antígeno epitelial humano; CA: antígeno carcinoide; } \\
\text { CEA: antígeno carcinoembrionario; CK: citoqueratina; ER: receptor de estrógeno; TTF: factor de transcripción tiroideo; WT-1: proteína } \\
\text { del tumor de Wilms. Fuente: Fernández-Antón Martínez MC, Parra-Blanco V, Avilés Izquierdo JA, Suárez-Fernández RM. Metástasis } \\
\text { cutáneas de origen visceral. Actas Dermosifiliogr. 2013;104(10):841-853. }\end{array}$} \\
\hline
\end{tabular}

\section{Diagnóstico Diferencial}


El diagnóstico diferencial de las metástasis cutáneas es muy amplio. Son diferenciales los tumores primariamente cutáneos (tanto benignos: dermatofibroma, granuloma piógeno, quiste epidérmico, tumores anexiales; como malignos: carcinoma basocelular o epidermoide, melanoma, carcinoma de células de Merkel, angiosarcoma), otras dermatosis (eccema, eritema anular centrífugo, eritema multiforme) y las vasculitis $(1-4,6,8)$.

\section{Pronóstico}

La presencia de metástasis cutáneas confieren un grave pronóstico, con una media de supervivencia de 3 a 6 meses, con escasas diferencias respecto a si las lesiones son únicas o múltiples. La mortalidad es superior al $70 \%$ en el primer año tras el diagnóstico. En relación a la localización, se establece una supervivencia media de 29 meses para metástasis locorregionales y 15,5 meses en caso de metástasis a distancia $(1,2,4)$.

\section{CONFLICTOS DE INTERÉS Y FUENTE DE FINANCIACIÓN}

Las autoras declaran no poseer conflictos de interés. Fuente de financiación: ninguna.

\section{REFERENCIAS BIBLIOGRÁFICAS}

1. Staiger H, Brau GF. Metástasis Cutáneas. Dermatol. Argent. 2013;19(1):15-25. URL.

2. Martínez Casimiro L, Vilata Corell JJ. Metástasis cutáneas de neoplasias internas. Med Cutan Iber Lat Am 2009;37(3):117-129. URL.

3. Colmenares LM, Zuluaga MA, Gallego P, Martínez WJ. Metástasis cutánea de un carcinoma de pulmón simulando un queratoacantoma. Rev CES Med 2013;27(1):107-112. URL.

4. Fernández-Antón Martínez MC, Parra-Blanco V, Avilés Izquierdo JA, Suárez-Fernández RM. Metástasis cutáneas de origen visceral. Actas Dermosifiliogr. 2013;104(10):841853. http://dx.doi.org/10.1016/i.ad.2012.06.005

5. De Siqueira VR, Frota AS, Maia IL, Queiroz HMC, Valença Júnior JT, Accioly Filho JW. Cutaneous involvement as the initial presentation of metastatic breast adenocarcinoma - Case report. An Bras Dermatol. 2014;89(6):960-963. http://dx.doi.org/10.1590/abd1806-4841.20142995

6. Gül U1, Kiliç A, Gönül M, Külcü Cakmak S, Erinçkan C. Spectrum of Cutaneous Metastases in 1287 Cases of Internal Malignancies: A Study from Turkey. Acta Derm Venereol. 2007;87(2):160-162. http://dx.doi.org/10.2340/00015555-0199

7. Martínez-Luna E, Puebla-Miranda M, Vega-Memije ME. Metástasis cutáneas de adenocarcinoma gástrico; informe de un caso. Caso Clínico. Rev Gastroenterol Mex, 2009;74(4):362-365. URL.

8. Nibhoria S, Kaur Tiwana K, Kaur M, Kumar S. A Clinicopathological and Immunohistochemical Correlation in Cutaneous Metastases from Internal Malignancies: A Five-Year Study. Journal of Skin Cancer 2014;793937:1-5. http://dx.doi.org/10.1155/2014/793937 